\title{
Reproducibility and validity of an FFQ developed for the Korea National Health and Nutrition Examination Survey (KNHANES)
}

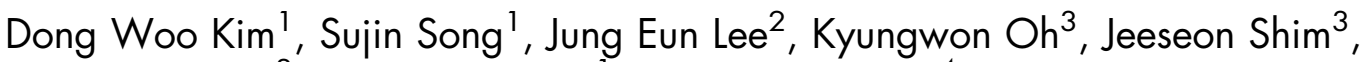 \\ Sanghui Kweon ${ }^{3}$, Hee Young Paik ${ }^{1}$ and Hyojee Joung ${ }^{4, *}$ \\ 'Department of Food and Nutrition, Seoul National University, Gwanak-gu, Seoul, Republic of Korea: ${ }^{2}$ Department \\ of Food and Nutrition, Sookmyung Women's University, Yongsan-gu, Seoul, Republic of Korea: ${ }^{3}$ Division of Health \\ and Nutrition Survey, Korea Centers for Disease Control and Prevention, Cheongwon-gun, Chungcheongbuk-do, \\ Republic of Korea: ${ }^{4}$ The Graduate School of Public Health and Institute of Health and Environment, Seoul National \\ University, 1 Gwanak-ro, Gwanak-gu, Seoul 151-742, Republic of Korea
}

Submitted 25 November 2013: Final revision received 1 July 2014: Accepted 10 July 2014: First published online 28 August 2014

\begin{abstract}
Objective: We aimed to evaluate the reproducibility and validity of the newly developed FFQ for the Korean National Health and Nutrition Examination Survey (KNHANES) and to estimate the measure's calibration factors.

Design: The 109-item FFQ was administered twice, approximately 9 months apart. We also collected four seasonal $3 \mathrm{~d}$ dietary records (DR) as a reference method. Correlation coefficients and joint classification were computed to compare intakes of energy, thirteen nutrients and eleven food groups between the two FFQ to evaluate reproducibility. For validity, de-attenuated and energy-adjusted correlation, joint classification and Bland-Altman statistics were calculated for energy and nutrients between the first FFQ and the DR. To calibrate the FFQ, we performed a linear regression analysis in which the DR were the dependent variables and FFQ, age and sex were the independent variables.

Setting: Seoul metropolitan area, Republic of Korea.

Subjects: A total of 126 adults aged 20-65 years.

Results: The average correlation coefficients measuring reproducibility were 0.54 for nutrients and 0.57 for food groups. The mean correlation coefficient measuring validity was 0.40 for all nutrients between the first FFQ and the DR. On average, $75 \%$ of the participants were classified into the same or adjacent quartiles, while $5 \%$ of the participants were grossly misclassified. The mean energy and nutrient intakes estimated by the calibrated FFQ were similar to the means estimated by the DR

Conclusions: The newly developed FFQ for assessing dietary intake in the KNHANES has acceptable reproducibility and modest validity compared with a $12 \mathrm{~d}$ DR collected over a 9-month period.
\end{abstract}

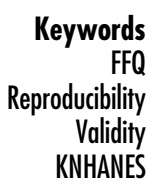

There has been increasing evidence that diet plays an important role in the development of various chronic diseases $^{(1)}$. Changes in the dietary patterns and nutritional status of Koreans due to economic growth and the introduction of Western lifestyles have caused significant changes in disease patterns in Korea ${ }^{(2)}$. This distinct nutritional transition in Korea, characterized by the coexistence of Westernized and traditional dietary patterns, can be explained by the speed of modernization in Korea compared with most other Asian countries ${ }^{(3)}$. Typical Korean dishes involve a mixture of various foods, cooking oils and seasonings ${ }^{(4)}$. Thus, collecting accurate estimates of dietary intake using instruments specifically designed for the Korean diet is essential for nutritional epidemiological studies that investigate the relationship between diet and disease among Koreans.

Although it is possible to quantify nutrient intake relatively accurately by $24 \mathrm{~h}$ recall $(24 \mathrm{HR})$ or dietary record $(\mathrm{DR})^{(5-7)}$, multiple measurements are necessary to estimate an individual's usual intake ${ }^{(8)}$. Because of the high cost and burden imposed on subjects to collect multiple dietary intake data, these methods may not be feasible for large-scale surveys, such as the Korea National Health and Nutrition Examination Survey (KNHANES). The FFQ has been used as an alternative method to measure long-term usual intake, but the FFQ must be developed and validated for specific target populations $^{(9-11)}$. 
Multiple $24 \mathrm{HR}$ or DR are often used in FFQ validation studies as a reference method for estimating usual nutrient intakes, as there is currently no gold standard method available. Calculating average nutrient intakes from multiple $24 \mathrm{HR}$ or DR can diminish within-individual variation, which can attenuate the association between dietary intake and disease risk. The number of days required to estimate usual intake depends on the type of nutrient being measured and the characteristics of the population ${ }^{(12,13)}$. Recently, validation studies of FFQ have adopted $2 \mathrm{~d}$ to $12 \mathrm{~d} 24 \mathrm{HR}$ or DR as reference methods ${ }^{(14-19)}$. A metaanalysis of forty articles on FFQ validation by Molag et $a l .{ }^{(20)}$ indicated that the correlation coefficients were significantly higher when the reference method was used for 8 to $14 \mathrm{~d}$ than for 1 to $7 \mathrm{~d}$.

The present study assessed the reproducibility and validity of a newly developed FFQ for KNHANES designed to estimate the usual nutrient intake of Korean adults and estimated the calibration factors of the FFQ with $12 \mathrm{~d}$ DR to correct raw intake data.

\section{Experimental methods}

\section{$\boldsymbol{F F Q}$}

The KNHANES is a nationwide survey designed by the Korea Centers for Disease Control and Prevention to assess the health and nutritional status of Koreans ${ }^{(21)}$. In 2007, the KNHANES became a continuous survey system using an integrated sampling method that increased the sample size ${ }^{(22)}$. The fourth KNHANES surveyed approximately 30000 individuals using a $1 \mathrm{~d} 24 \mathrm{HR}$ and a simple FFQ to measure dietary intake during 2007 to 2009.

A new FFQ was developed in 2009 by the KNHANES team of the Korea Centers for Disease Control and Prevention based on $1 \mathrm{~d} 24 \mathrm{HR}$ data collected from adults aged $>19$ years in the fourth KNHANES (2007-2009). The procedure for developing the FFQ has been described elsewhere ${ }^{(23)}$. Briefly, the food and dish items that contributed $90 \%$ of the energy and thirteen nutrients (carbohydrates, protein, fat, $\mathrm{Ca}, \mathrm{P}, \mathrm{Fe}, \mathrm{Na}, \mathrm{K}$, vitamin $\mathrm{A}$, thiamin, riboflavin, niacin and vitamin C) or explained $90 \%$ of the between-individual variation were selected. Food items that were consumed by less than $1 \%$ of the total population were excluded. Similar food and dish items were combined to reduce the number of items. Ultimately, the FFQ list consisted of 109 food and dish items. The response categories for the frequency of consumption during the previous year were divided into nine levels: none, 1 time/month, 2-3 times/month, 1 time/week, 2-4 times/week, 4-6 times/week, 1 time/d, 2 times/d and $\geq 3$ times/d. Participants were also asked to select one of three portion sizes: small (0.5), medium (1.0) and large $(1 \cdot 5-2 \cdot 0)$, which were determined based on the distribution of portion sizes from the dietary data in the fourth KNHANES.

The nutrient database used to calculate nutrient intake for the present study was the seventh edition of the food composition table from the Korean National Academy of Agricultural Science ${ }^{(24)}$. The database consists of 5112 food items and provides energy and nutrient values for each of the foods listed. To calculate the daily nutrient intakes, the reported FFQ frequency data were coded as daily frequencies. For example, the $2-4$ times/week option was transformed into 0.43 times/d, as estimated by the formula $(2+4) /(2 / 7)$.

\section{Participants and study design}

A convenience sample of 140 healthy adults (aged 20-65 years) residing in the Seoul metropolitan area participated in the study. The sample size was determined based on the minimum correlation coefficient for nutrient intake between the FFQ and $12 \mathrm{~d}$ DR in previous reports ${ }^{(25)}$. For twelve replicates of daily intake, a sample size of 110 persons was estimated to be sufficient to maintain constant precision in the correlation coefficient (assuming a correlation of 0.5 with a variance of 0.14 ) between the FFQ and the average of multiple $\mathrm{DR}^{(25)}$. Assuming a potential dropout rate of $20 \%$ during the follow-up period, the final recruited sample size was 140 .

Among the 140 participants, 126 (90\%) completed both FFQ and $12 \mathrm{~d}$ DR. The first FFQ (FFQ1) and the first $3 \mathrm{~d}$ DR were administered by an interviewer in August 2009. Three additional $3 \mathrm{~d}$ DR were conducted every three months, and the second FFQ (FFQ2) was conducted in May 2010 to evaluate the reproducibility of the FFQ. Demographic information, including sex and age, was collected from the participants using a structured questionnaire. The study was conducted according to the guidelines in the Declaration of Helsinki and all procedures involving human subjects were approved by the Institutional Review Board of Seoul National University on 20 July 2009. Written informed consent was obtained from all participants.

\section{Collection of dietary records}

The $3 \mathrm{~d}$ DR were completed four times during the 9-month period following the administration of FFQ1 to account for seasonal variations in consumption. The participants were asked to write down every food and dish consumed on three non-consecutive days, including two weekdays and one weekend day. Detailed guidelines, including visual two-dimensional household measurement tools, were distributed to the participants to assist them in completing the DR when FFQ1 was conducted. To improve the quality of the DR, the records were reviewed by nutritionists and missing values or vague answers were clarified by telephone interview. The same nutrient database that was used for the FFQ was used to calculate individual nutrient intakes from the $12 \mathrm{~d}$ DR. In addition, the standard recipe database used for the KNHANES was applied to convert the reported dishes into constituent foods. A total of 3596 dishes were included in the standard recipe database, which provided a list of food items and standard weights of each food in a dish. 


\section{Calculation of nutrient and food group intakes}

Means and standard deviations were calculated for total energy and thirteen nutrients (protein, fat, carbohydrates, $\mathrm{Ca}, \mathrm{P}, \mathrm{Fe}, \mathrm{K}, \mathrm{Na}$, vitamin $\mathrm{A}$, thiamin, riboflavin, niacin and vitamin C) in each of the FFQ and $12 \mathrm{~d}$ DR. Means and standard deviations of intake for eleven food groups (rice; noodles and dumplings; breads and rice cakes; soups and stews; soyabeans, eggs, meat and fish; vegetables, seaweed and potatoes; milk and dairy products; fruits; beverages; snacks; alcoholic beverages) were also calculated. Log (natural) transformations were performed before statistical analysis to improve the normality of the distribution of the crude data.

Data from the $12 \mathrm{~d}$ DR were used to estimate withinand between-individual variances according to the Multiple Source Method and de-attenuation was computed according to the equation from Willett ${ }^{(25)}$. Subsequently, the calculations of nutrient intake were adjusted for total energy intake using the residual method ${ }^{(25)}$ for both the FFQ and the $12 \mathrm{~d}$ DR (energy-adjusted data).

\section{Reproducibility}

Reproducibility was evaluated by comparing the data on energy intake, intakes of thirteen nutrients and intakes of eleven food groups from FFQ1 and FFQ2. Because the log-transformed data showed a normal distribution for energy and all nutrients of FFQ1 and FFQ2, Pearson correlation coefficients were used to compare FFQ1 and FFQ2 as a measure of reproducibility ${ }^{(26)}$. On the other hand, the log-transformed data did not show a normal distribution for the majority of the food group intake data; therefore, Spearman correlation coefficients were used to compare the food group intake data. We also calculated a ratio of the geometric means to estimate changes in mean intake levels between FFQ1 and FFQ2 ${ }^{(27)}$, and the ratios were multiplied by 100 to be expressed as a percentage, with $100 \%$ representing an ideal agreement ${ }^{(28)}$. To evaluate the joint classification, the intakes of nutrients and food groups estimated by FFQ1 and FFQ2 were classified into quartiles. The percentage of participants classified in the same quartile, in adjacent quartiles and in opposite quartiles by the two FFQ was analysed.

\section{Validity}

The validity of the FFQ was assessed by comparing the average intake estimates from the $12 \mathrm{~d}$ DR with those from FFQ1. FFQ1 was used instead of FFQ2 to limit possible bias due to a training effect ${ }^{(27)}$ and to replicate typical administration of the FFQ where participants have no experience quantifying their dietary intake. Validation of the FFQ in the present study used three methods: Pearson correlation coefficients, joint classification analysis and the Bland-Altman method ${ }^{(29)}$. Pearson correlation and joint classification analysis were conducted in the same manner as described for the reproducibility analysis. To apply the Bland-Altman method, for each nutrient and participant, the difference in intake between the two methods and the average intake of the two methods (FFQ1 and $12 \mathrm{~d}$ DR) were calculated and plotted. Because the exponential of a difference is a ratio, we calculated the mean difference (given as a percentage) and the limits of agreement (LOA). The LOA were calculated using the following equation:

$$
\begin{aligned}
\mathrm{LOA}= & \text { mean difference } \pm 1.96 \\
& \times \text { standard deviation of the difference. }
\end{aligned}
$$

The LOA ranged from $50 \%$ to $200 \%$, which indicates that $95 \%$ of participants' FFQ data were between half and two times their $12 \mathrm{~d}$ DR data. Therefore, in the present study, we considered LOA between $50 \%$ and $200 \%$ suitable $_{(30)}$.

\section{Calibration}

The FFQ was calibrated using multivariate linear regression associating the data obtained from the $12 \mathrm{~d}$ DR (dependent variable) with the data obtained from FFQ1 (independent variable). The regression model also included sex and age as independent variables. The regression intercepts $(\alpha)$, regression line slopes $(\lambda)$ and $95 \%$ confidence interval were estimated. Calibrated values (mean and standard deviation) for energy and each nutrient were calculated based on $\lambda$ and $\alpha$ using following equation:

$$
\text { Calibrated FFQ }=\alpha+\lambda_{1}(\mathrm{FFQ} 1)+\lambda_{2}(\operatorname{sex})+\lambda_{3}(\text { age }) .
$$

The statistical software package SAS for Windows version $9 \cdot 2$ was used for all statistical analyses. Statistical significance was determined based on $P$ values $(<0.05)$ and $95 \%$ confidence intervals.

\section{Results}

The study group consisted of sixty-three men (50.0\%) and sixty-three women (50.0\%) between 20 and 65 years of age, while the 2007 KNHANES was conducted with adults between 20 and 103 years of age (40.9\% male). The mean age of participants in the present validation study was $42 \cdot 7$ (sD 13.1) years, while the 2007 KNHANES sample had a mean age of $50 \cdot 0$ (SD 16.6) years. There were significant differences in the distribution of participants by age and gender between the current sample and the KNHANES sample.

Table 1 shows the reproducibility of the FFQ over a 9-month period (FFQ1 $v$. FFQ2). The average energy and nutrient intakes from the FFQ2 were somewhat lower than those from FFQ1 (percentage agreement ranging from $103 \%$ to $110 \%)$. The Pearson correlation coefficients for nutrients between FFQ1 and FFQ2 ranged from 0.53 to 0.62 for crude intake data (mean $r=0.56$ ). After adjusting nutrient intake for total energy intake, the correlation coefficients for nutrients (mean $r=0.55$ ) increased for some nutrients (fat, carbohydrate, Ca, P, K and vitamin C), but decreased for others. For the food groups, Spearman correlation coefficients between FFQ1 and FFQ2 ranged 
Table 1 Daily energy, nutrient and food intakes measured by the first and second administration of the FFQ and the reproducibility between FFQ administrations among Korean adults $(n$ 126) aged 20-65 years residing in the Seoul metropolitan area, August 2009-May 2010

\begin{tabular}{|c|c|c|c|c|c|c|c|c|c|c|c|}
\hline & \multicolumn{2}{|c|}{ FFQ1 } & \multicolumn{2}{|c|}{ FFQ2 } & \multicolumn{2}{|c|}{ Ratio* } & \multicolumn{2}{|c|}{ Correlation† } & \multicolumn{3}{|c|}{ Quartile classification (\%) } \\
\hline & Mean & SD & Mean & SD & Mean & $95 \% \mathrm{Cl}$ & Crude & $\begin{array}{l}\text { Energy- } \\
\text { adjusted }\end{array}$ & Same & $\begin{array}{l}\text { Same }+ \\
\text { adjacent }\end{array}$ & Opposite \\
\hline \multicolumn{12}{|l|}{ Energy/nutrients $\ddagger$} \\
\hline Energy (kJ) & $9547 \cdot 7$ & 3236.5 & $9160 \cdot 9$ & $3319 \cdot 8$ & 105 & 99,111 & 0.54 & - & 44 & 83 & 2 \\
\hline Energy (kcal) & 2281.9 & 773.5 & 2189.5 & 793.4 & 105 & 99,111 & 0.54 & - & 44 & 83 & 2 \\
\hline Protein (g) & 80.7 & 30.8 & $76 \cdot 7$ & 33.3 & 106 & 100,114 & 0.55 & 0.52 & 50 & 84 & 2 \\
\hline Fat $(\mathrm{g})$ & $50 \cdot 1$ & 23.5 & 47.5 & $26 \cdot 9$ & 110 & 101,119 & 0.62 & 0.65 & 51 & 87 & 2 \\
\hline Carbohydrate $(\mathrm{g})$ & 360.9 & 128.4 & 347.6 & 117.9 & 103 & 98,110 & 0.54 & 0.61 & 44 & 83 & 4 \\
\hline $\mathrm{Ca}(\mathrm{mg})$ & 636.4 & 260.5 & 589.4 & 259.1 & 108 & 101,117 & 0.58 & 0.63 & 46 & 83 & 2 \\
\hline$P(\mathrm{mg})$ & $1369 \cdot 7$ & $472 \cdot 6$ & $1303 \cdot 3$ & $486 \cdot 2$ & 106 & 99,112 & 0.53 & 0.61 & 45 & 80 & 3 \\
\hline $\mathrm{Fe}(\mathrm{mg})$ & $16 \cdot 4$ & 6.9 & $15 \cdot 7$ & $7 \cdot 0$ & 105 & 98,113 & 0.57 & 0.51 & 41 & 82 & 4 \\
\hline $\mathrm{K}(\mathrm{mg})$ & $3771 \cdot 1$ & $1611 \cdot 8$ & $3491 \cdot 1$ & $1427 \cdot 4$ & 107 & 101,114 & 0.57 & 0.65 & 47 & 84 & 1 \\
\hline $\mathrm{Na}(\mathrm{mg})$ & 5633.7 & $2543 \cdot 6$ & $4965 \cdot 3$ & 2392.0 & 114 & 106,123 & 0.60 & 0.35 & 45 & 87 & 2 \\
\hline Vitamin A ( $\mu \mathrm{g} R E)$ & $824 \cdot 1$ & 384.7 & $750 \cdot 5$ & 367.5 & 110 & 101,120 & 0.55 & 0.44 & 44 & 83 & 2 \\
\hline Thiamin (mg) & 1.58 & 0.62 & 1.49 & 0.65 & 107 & 100,115 & 0.55 & 0.48 & 44 & 83 & 2 \\
\hline Riboflavin (mg) & 1.33 & 0.54 & 1.26 & 0.55 & 107 & 100,115 & 0.55 & 0.55 & 43 & 87 & 2 \\
\hline Niacin (mg) & $18 \cdot 9$ & $7 \cdot 1$ & $18 \cdot 1$ & $7 \cdot 7$ & 106 & 99,113 & 0.54 & 0.45 & 50 & 83 & 3 \\
\hline Vitamin C (mg) & $139 \cdot 6$ & 82.9 & $123 \cdot 3$ & $60 \cdot 2$ & 110 & 101,119 & 0.61 & 0.64 & 48 & 87 & 3 \\
\hline \multicolumn{12}{|l|}{ Food groups $(\mathrm{g}) \S$} \\
\hline Rice & 265.3 & 105.5 & 263.2 & $105 \cdot 7$ & 101 & 92,110 & 0.33 & 0.38 & 33 & 71 & 4 \\
\hline Noodles and dumplings & $82 \cdot 0$ & 84.5 & 70.5 & $70 \cdot 7$ & 117 & 96,143 & 0.69 & 0.49 & 46 & 90 & 1 \\
\hline Breads and rice cakes & $35 \cdot 9$ & $39 \cdot 1$ & 43.9 & $50 \cdot 6$ & 73 & 58,93 & 0.58 & 0.58 & 48 & 84 & 2 \\
\hline Soups and stews & 108.4 & 78.2 & $96 \cdot 7$ & 69.8 & 115 & 99,133 & 0.48 & 0.34 & 44 & 79 & 5 \\
\hline $\begin{array}{l}\text { Soyabeans, eggs, meat } \\
\text { and fish }\end{array}$ & $183 \cdot 1$ & $122 \cdot 8$ & $169 \cdot 4$ & $134 \cdot 1$ & 116 & 103,131 & 0.61 & 0.55 & 44 & 88 & 3 \\
\hline $\begin{array}{l}\text { Vegetables, seaweed and } \\
\text { potatoes }\end{array}$ & $330 \cdot 1$ & $228 \cdot 0$ & $280 \cdot 3$ & $176 \cdot 4$ & 115 & 102,129 & 0.55 & 0.52 & 45 & 83 & 2 \\
\hline Milk and dairy products & 192.5 & 185.9 & 195.4 & $186 \cdot 2$ & 97 & 75,126 & 0.67 & 0.71 & 57 & 91 & 2 \\
\hline Fruits & 233.4 & $236 \cdot 3$ & $222 \cdot 9$ & 187.9 & 95 & 80,112 & 0.71 & 0.71 & 54 & 89 & 2 \\
\hline Beverages & 140.4 & 163.0 & $117 \cdot 8$ & 136.6 & 127 & 104,155 & 0.62 & 0.60 & 51 & 87 & 4 \\
\hline Snacks & $19 \cdot 2$ & $25 \cdot 2$ & $20 \cdot 3$ & 35.5 & 117 & 85,162 & 0.57 & 0.52 & 43 & 83 & 2 \\
\hline Alcoholic beverages & $145 \cdot 6$ & 231.9 & $157 \cdot 8$ & 340.3 & 105 & 87,126 & 0.87 & 0.87 & 67 & 98 & 0 \\
\hline
\end{tabular}

FFQ1, FFQ administered at baseline; FFQ2, FFQ administered 9 months after FFQ1; RE, retinol equivalents.

${ }^{*}$ Geometric mean of individual ratios between intakes as recorded by FFQ1 and FFQ2.

†All coefficients were significant.

‡Pearson correlation coefficients estimated from log-transformed intakes (crude) and energy-adjusted intakes by the residual method as estimated by FFQ1 and FFQ2.

$\S S$ pearman correlation coefficients estimated from log-transformed intakes (crude) and energy-adjusted intakes by the residual method as estimated by FFQ1 and FFQ2.

from 0.33 (rice) to 0.87 (alcoholic beverages) for crude intake data, and energy-adjusted correlation coefficients were higher for alcoholic beverages, fruit and milk and dairy products $(0.87,0.71$ and 0.71 , respectively) than for other food groups. Joint classification into quartiles mostly showed good agreement (same + adjacent quartile) for nutrient and food group categories (approximately $80 \%$ ); the highest proportions of participants correctly classified into the same intake quartile were observed for fat (51\%) and alcoholic beverages (67\%).

Table 2 shows the validity of the FFQ1 in terms of Pearson correlation coefficients, joint classification into quartiles and the results of the Bland-Altman procedure. The significant Pearson correlation coefficients between FFQ1 and the $12 \mathrm{~d}$ DR ranged from 0.27 to 0.45 using the crude data (mean $r=0.38$ ) and from 0.22 to 0.64 using the energy-adjusted data (mean $r=0 \cdot 41$ ). Adjusting for total energy intake, the correlations for eight nutrients such as fat, carbohydrate, $\mathrm{Ca}, \mathrm{P}, \mathrm{Fe}, \mathrm{K}$, riboflavin and vitamin $\mathrm{C}$ were improved. In the joint-classification analysis (Table 2), 63\% (thiamin) to $86 \%$ (carbohydrate) of participants were classified into the same or adjacent quartile groups for FFQ1 and $12 \mathrm{~d}$ DR (mean $=75 \%$ ). The proportion grossly misclassified (i.e. ranked in the lowest or highest quartile of the FFQ1 data but in the opposite quartile of the $12 \mathrm{~d} D R)$ was small $(1-10 \%$, mean $=5 \%)$.

Table 2 also shows the coefficients of the calibration analysis. For all nutrients except for thiamin, the linear calibration coefficients for the slope estimates (linear regression of $12 \mathrm{~d}$ DR $v$. FFQ1) were significant. The means from the calibrated FFQ were similar to those from the $12 \mathrm{~d}$ DR, but the calibrated FFQ had reduced standard deviation estimates for energy and most nutrients (data not shown).

The mean agreement between FFQ1 and the $12 \mathrm{~d}$ DR ranged from $73 \%$ for vitamin A to $116 \%$ for carbohydrate. The LOA for almost all nutrients ranged between $50 \%$ and $200 \%$, except for energy, vitamin A and vitamin C. 
Table 2 Daily energy and nutrient intakes estimated by the $12 \mathrm{~d}$ dietary record (DR) and the validity between the $12 \mathrm{~d}$ DR and the first administration of the FFQ among Korean adults $(n$ 126) aged 20-65 years residing in the Seoul metropolitan area, August 2009-May 2010

\begin{tabular}{|c|c|c|c|c|c|c|c|c|c|c|c|}
\hline \multirow[b]{2}{*}{ Energy/nutrients } & \multicolumn{2}{|c|}{$12 \mathrm{~d}$ DR } & \multicolumn{2}{|c|}{ Pearson correlation } & \multicolumn{3}{|c|}{ Quartile classification (\%) } & \multicolumn{2}{|c|}{ Mean agreement (\%) } & \multicolumn{2}{|c|}{ Calibration factor } \\
\hline & Mean & SD & Crude & Adjusted* & Same & $\begin{array}{l}\text { Same + } \\
\text { adjacent }\end{array}$ & Opposite & Mean & 95 \% LOA† & $\lambda_{1}$ value $\ddagger$ & $95 \% \mathrm{Cl}$ \\
\hline Energy $(\mathrm{kJ})$ & 8411.7 & $1930 \cdot 6$ & 0.43 & - & 35 & 78 & 5 & 109 & 58,205 & 0.191 & $0.097,0.285$ \\
\hline Energy (kcal) & $2010 \cdot 5$ & 461.4 & 0.43 & - & 35 & 78 & 5 & 109 & 58,205 & $0 \cdot 191$ & $0.097,0.285$ \\
\hline Protein (g) & 78.9 & $21 \cdot 1$ & 0.37 & 0.33 & 31 & 67 & 6 & 98 & 73,32 & 0.213 & $0.114,0.312$ \\
\hline Fat (g) & 51.9 & $16 \cdot 4$ & 0.45 & 0.54 & 43 & 81 & 2 & 89 & 54,148 & 0.176 & $0.092,0.260$ \\
\hline Carbohydrate (g) & 299 & 67 & 0.44 & 0.64 & 40 & 86 & 1 & 116 & 95,42 & 0.444 & $0.326,0.562$ \\
\hline $\mathrm{Ca}(\mathrm{mg})$ & 588 & 169 & 0.43 & 0.48 & 40 & 82 & 3 & 102 & 58,179 & 0.231 & $0.147,0.316$ \\
\hline $\mathrm{P}(\mathrm{mg})$ & 1262 & 315 & 0.37 & 0.45 & 36 & 81 & 4 & 105 & 82,134 & 0.315 & $0.202,0.42 \mathrm{c}$ \\
\hline $\mathrm{Fe}(\mathrm{mg})$ & $16 \cdot 3$ & 5.5 & 0.36 & 0.38 & 33 & 75 & 6 & 96 & 60,153 & 0.186 & $0.077,0.296$ \\
\hline $\mathrm{K}(\mathrm{mg})$ & 3335 & 904 & 0.40 & 0.53 & 37 & 81 & 3 & 107 & 71,162 & 0.280 & $0.188,0.371$ \\
\hline $\mathrm{Na}(\mathrm{mg})$ & 5877 & 2087 & 0.36 & 0.23 & 26 & 72 & 10 & 91 & 50,166 & $0 \cdot 151$ & $0.028,0.274$ \\
\hline Vitamin A ( $\mu \mathrm{g} R E)$ & 1083 & 510 & 0.27 & 0.22 & 26 & 66 & 10 & 73 & 35,154 & $0 \cdot 138$ & $0.012,0.264$ \\
\hline Thiamin (mg) & 1.47 & 0.45 & 0.39 & $0.15 \S$ & 22 & 63 & 8 & 103 & 68,155 & 0.091 & $-0.028,0.210$ \\
\hline Riboflavin (mg) & 1.46 & 0.44 & 0.37 & 0.38 & 35 & 75 & 8 & 87 & 58,132 & 0.236 & $0.129,0.343$ \\
\hline Niacin (mg) & $18 \cdot 6$ & $5 \cdot 0$ & 0.29 & 0.29 & 33 & 67 & 6 & 98 & 71,135 & 0.215 & $0.086,0.345$ \\
\hline Vitamin C (mg) & 117 & 43 & 0.37 & 0.47 & 36 & 80 & 6 & 108 & 52,224 & 0.258 & $0.158,0.357$ \\
\hline
\end{tabular}

LOA, limit of agreement; RE, retinol equivalents.

${ }^{*}$ Natural log-transformed, de-attenuated and energy-adjusted intake of nutrients by the Multiple Source Method.

TLOA determined as the mean difference $+1.96 \times$ SD of the difference.

¥Natural log-transformed energy intake estimated from the FFQ1 (independent variable) and from the mean of $12 \mathrm{~d}$ DR (dependent variable).

All coefficients were significant except those marked with §.

The narrowest LOA was found for carbohydrate and the widest LOA was found for vitamin C. Figure 1 illustrates the Bland-Altman plots for carbohydrate and vitamin A, the nutrients with the best and worst validity results, respectively. A regression analysis (linear regression of the difference between the two methods $v$. the average of the two methods) indicated a significant linear trend for both nutrients (slope $=0.277$ for carbohydrate and 0.431 for vitamin A), implying that agreement between the methods varied according to the magnitude of intake.

For validity of the FFQ compared with the $12 \mathrm{~d}$ DR, the correlations of energy intake and intake of most nutrients were higher in females (mean $r=0.45$ ) compared with males (mean $r=0.30$ ) using the energy-adjusted data (Table 3). The correlation coefficients for protein, Na, vitamin A, thiamin, riboflavin and niacin were not significant in males, but the correlation coefficients for energy intake and intake of all nutrients except niacin were significant in females. Among the participants aged 20-45 years (mean $r=0 \cdot 46$ ), the energy-adjusted correlations were higher for most nutrients compared with the higher age group aged 46-65 years (mean $r=0 \cdot 28$ ), except for three macronutrients.

The validity was also evaluated with FFQ2 and the mean of FFQ1 and FFQ2 compared with the $12 \mathrm{~d}$ DR (see online supplementary material, Supplemental Table 1). The Pearson correlation coefficients between FFQ2 and $12 \mathrm{~d}$ DR ranged from 0.21 for thiamin to 0.61 for fat (mean $r=0.38$ for energy-adjusted data), and those between the mean of FFQ1 and FFQ2 and $12 \mathrm{~d}$ DR ranged from $0 \cdot 20$ for thiamin to 0.69 for carbohydrate (mean $r=0.43$ for energy-adjusted data). The average correlation coefficient found in comparing the mean of FFQ1 and FFQ2 with $12 \mathrm{~d}$ DR was similar to that found in comparing FFQ1 with $12 \mathrm{~d}$ DR, but the validity assessed with FFQ2 was slightly lower than that found with FFQ1 and the mean of FFQ1 and FFQ2.

\section{Discussion}

The purpose of the present study was to assess the reproducibility and validity of the newly developed FFQ for estimating usual dietary intake in KNHANES using $12 \mathrm{~d}$ $\mathrm{DR}$ as the reference method. We found acceptable reproducibility between FFQ1 and FFQ2, which were conducted over a 9-month period, and modest validity of FFQ compared with the $12 \mathrm{~d}$ DR. The validity of the FFQ was slightly higher in females and younger participants compared with males and older participants.

The interval between the administration of FFQ1 and FFQ2 was an important factor in the design of the present FFQ validation study, as it was important to avoid seasonal variation and to stay within the period defined by the $\mathrm{FFQ}^{(31)}$. If the interval was too short, the second FFQ might have been influenced by the participants' memory of the first FFQ and thus the reproducibility would be overestimated $^{(32)}$. In contrast, if the interval were too long, the dietary patterns of individuals may have changed, leading to an underestimation of the reproducibility ${ }^{(33)}$. Although the time intervals used in other studies varied from $2 \mathrm{~h}$ to 15 years $^{(34)}$, we chose a time interval of almost one year because dietary intake among Koreans has shown significant seasonal changes ${ }^{(35)}$. In the present 

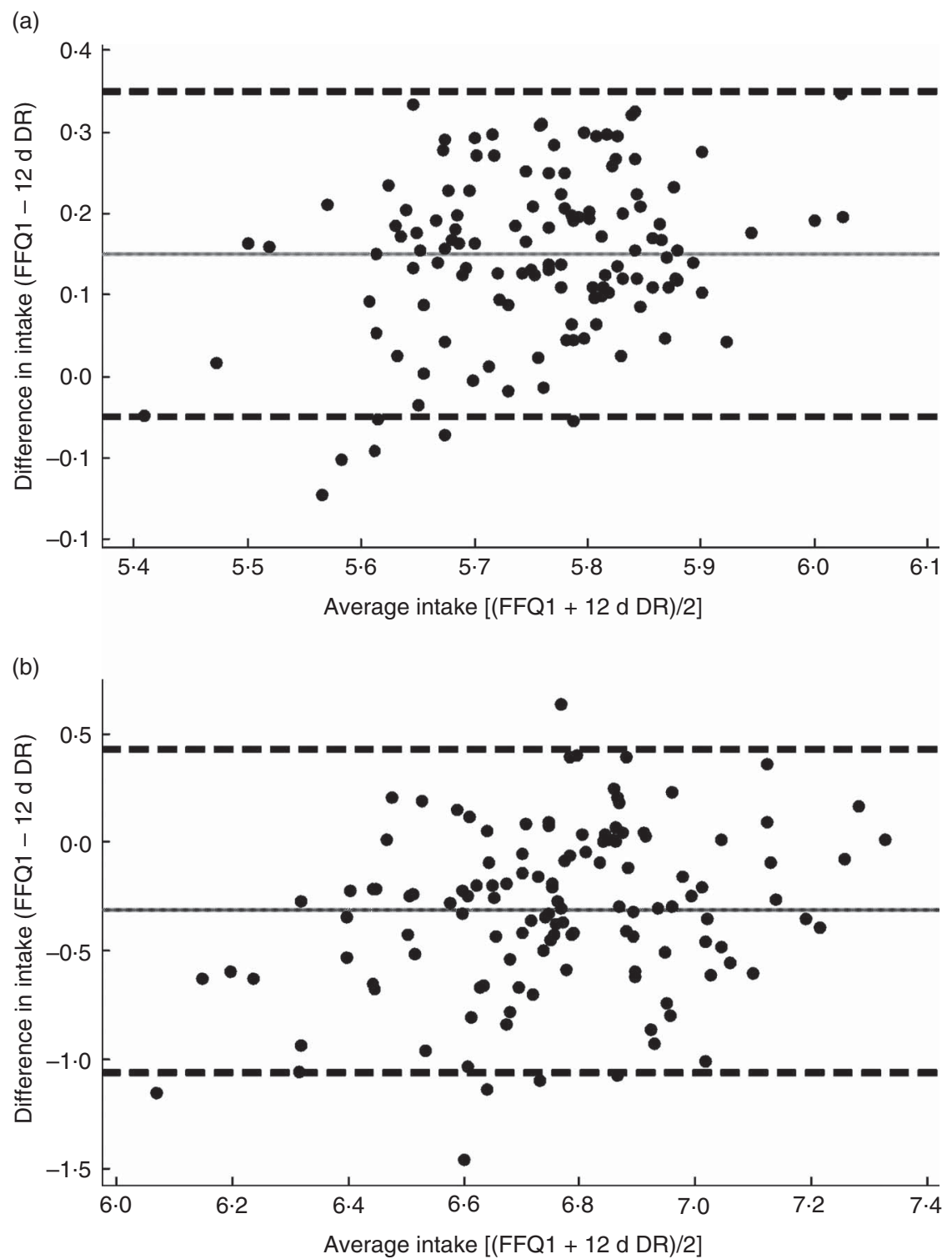

Fig 1 Bland-Altman plots showing the relationship between the differences in daily intake of (a) carbohydrate and (b) vitamin A estimated by the first administration of the FFQ (FFQ1) and the $12 \mathrm{~d}$ dietary record (DR) and the corresponding mean daily intakes estimated by the two methods among Korean adults $(n$ 126) aged 20-65 years residing in the Seoul metropolitan area, August 2009-May 2010. _ indicates the mean difference and - - - indicate the $95 \%$ limits of agreement between FFQ1 and the $12 \mathrm{~d}$ DR in natural log scale

study, a significant difference in mean nutrient intake was not observed for nutrients except fat, riboflavin, niacin and vitamin $\mathrm{C}$ between the first $3 \mathrm{~d}$ DR and the fourth $3 \mathrm{~d}$ DR (see online supplementary material, Supplemental Table 2); therefore we can assume that the dietary intake of individuals was not significantly different over the 9-month period. The range of correlation coefficients for reproducibility in our study was similar to those reported in previous studies that have been conducted using the same time interval in Western countries ${ }^{(27)}$ and Korea $(0.50<r<0.60)^{(36)}$.

The correlation coefficients between FFQ1 and the $12 \mathrm{~d}$ DR were high for macronutrients such as fat and carbohydrates and were low for vitamins after adjusting for total energy intake. Similarly, the proportion of agreement in exact and adjacent quartiles using jointclassification analysis was higher for fat and carbohydrate (i.e. above $80 \%$ ) and relatively low for vitamins, ranging from $67 \%$ to $79 \%$. Similar to the correlation coefficients, the joint classification indicated that the FFQ adequately ranked participants according to their intakes of fat and carbohydrates; however, the intakes of vitamin A and thiamin (percentage of same plus adjacent quartiles $<70 \%)$ were not well classified by the FFQ. Another recent FFQ validation study conducted in Korea reported that correlations between macronutrient measurements 
Table 3 Pearson correlation coefficients between daily energy and nutrient intakes estimated with the first administration of the FFQ and the $12 \mathrm{~d}$ dietary record according to gender and age group among Korean adults $(n 126)$ aged $20-65$ years residing in the Seoul metropolitan area, August 2009-May 2010

\begin{tabular}{|c|c|c|c|c|c|c|c|c|}
\hline \multirow[b]{3}{*}{ Energy/nutrients } & \multicolumn{4}{|c|}{ Sex } & \multicolumn{4}{|c|}{ Age group } \\
\hline & \multicolumn{2}{|c|}{ Men $(n$ 63) } & \multicolumn{2}{|c|}{ Women ( $n$ 63) } & \multicolumn{2}{|c|}{$20-45$ years $(n 63)$} & \multicolumn{2}{|c|}{$46-65$ years $(n 63)$} \\
\hline & Crude & Energy-adjusted* & Crude & Energy-adjusted ${ }^{*}$ & Crude & Energy-adjusted* & Crude & Energy-adjusted ${ }^{\star}$ \\
\hline Energy & 0.31 & - & 0.35 & - & 0.48 & - & 0.39 & - \\
\hline Protein & $0.22 \dagger$ & $0.13 \dagger$ & 0.35 & 0.53 & 0.41 & 0.31 & 0.33 & 0.38 \\
\hline Fat & 0.37 & $0.44^{\circ}$ & 0.44 & 0.63 & 0.39 & 0.36 & 0.38 & 0.40 \\
\hline Carbohydrate & 0.36 & 0.61 & 0.44 & 0.64 & 0.49 & 0.44 & 0.36 & 0.72 \\
\hline $\mathrm{Ca}$ & 0.38 & 0.36 & 0.48 & 0.53 & 0.51 & 0.66 & 0.35 & $0.21 \dagger$ \\
\hline $\mathrm{P}$ & 0.28 & 0.39 & 0.38 & 0.52 & 0.43 & 0.59 & 0.32 & 0.27 \\
\hline $\mathrm{Fe}$ & 0.28 & 0.32 & 0.39 & 0.38 & 0.42 & 0.54 & 0.27 & $0.07 \dagger$ \\
\hline $\mathrm{K}$ & 0.34 & 0.46 & 0.44 & 0.54 & 0.47 & 0.66 & 0.30 & 0.39 \\
\hline $\mathrm{Na}$ & $0.22 \dagger$ & $0.17 \dagger$ & 0.36 & 0.32 & 0.33 & $0.23 \dagger$ & 0.39 & $0.21 \dagger$ \\
\hline Vitamin A & 0.25 & $0.14 \dagger$ & 0.28 & $0.19 \dagger$ & 0.35 & 0.45 & $0.24 \dagger$ & $0.06 \dagger$ \\
\hline Thiamin & $0.23 \dagger$ & $0.04 \dagger$ & 0.41 & 0.27 & 0.49 & 0.23 & 0.28 & $0.09 \dagger$ \\
\hline Riboflavin & 0.28 & $0.16 \dagger$ & 0.41 & 0.52 & 0.45 & 0.52 & 0.33 & 0.31 \\
\hline Niacin & $0.23 \dagger$ & $0.24 \dagger$ & $0.24 \dagger$ & 0.30 & 0.35 & 0.42 & $0.25 \dagger$ & $0.21 \dagger$ \\
\hline Vitamin C & 0.30 & 0.39 & 0.45 & 0.47 & 0.45 & 0.59 & 0.26 & 0.35 \\
\hline
\end{tabular}

*Natural log-transformed, de-attenuated and energy-adjusted intake of nutrients by the Multiple Source Method.

All coefficients were significant except those marked with $\dagger$.

were high, but those for micronutrients were relatively low, which was similar to our results ${ }^{(37)}$.

The correlations between FFQ1 and the $12 \mathrm{~d}$ DR in our study were somewhat lower than those reported in Western countries ${ }^{(27,32)}$ but were comparable to other Korean studies ${ }^{(36-39)}$. In 2010, a validation study ( $n$ 85, age 33-70 years) conducted in Korea reported that correlation coefficients between FFQ and $3 \mathrm{~d}$ DR ranged from $0 \cdot 27(\mathrm{Fe})$ to 0.60 (carbohydrate) $^{(37)}$. Another validation study conducted with a relatively large sample size and many days of DR ( $n$ 288, $12 \mathrm{~d}$ DR, aged over 30 years) reported that the median correlation coefficients were $0 \cdot 31$ for nutrients and 0.29 for foods ${ }^{(36)}$, which were similar to ours. On the other hand, a recent validation study ( $n$ 305, $3 \mathrm{~d}$ DR, age 38-78 years) in Korean adults reported that the median correlation coefficient value was 0.55 and more than $75 \%$ of the participants were classified into the same or adjacent quartiles ${ }^{(40)}$, which were slightly higher than ours, most likely because participants were recruited from a health promotion centre following a medical check-up; therefore, participants may have been more concerned about their health or food intake.

The reasons for lower validity in Korea than in Western countries may be related to Koreans' eating habits, which include sharing main and side dishes with multiple people. This difference may negatively influence Koreans' ability to report consumption frequency and portion sizes. Moreover, averaging the amount of intake over almost one year may lead to over- or underestimation using the FFQ. Another traditional measurement error for the FFQ items that contained multiple foods and dishes can be noted. Those food groups composed of only one food or dish item ('alcoholic beverages', 'fruits' and 'milk and dairy products') showed higher correlation coefficients for reproducibility (above 0.70) compared with those composed of multiple foods.

Correlation coefficients have been widely used in FFQ validation studies; however, they can assess the degree of relative association between two methods, but not the degree of agreement ${ }^{(29)}$. Thus, joint classification and the Bland-Altman method were applied in the present study to assess agreement. The classification of participants by nutrient intake level is often useful for comparing disease risk across categories of intake in epidemiological studies. In our study, the mean percentage of participants classified into the same or adjacent quartiles by the two methods was approximately $75 \%$, a level comparable to those reported in previous studies ${ }^{(41-43)}$. In addition, the percentage of participants classified into the opposite quartile tended to be low. Our results showed that the FFQ is acceptable for classifying participants' nutrient intake, which may be useful for studying diet-disease relationships. Using the Bland-Altman method, there was no systematic bias for most of the nutrients and the dispersion estimated by the Bland-Altman procedure suggested good concordance, except for energy, vitamin A and vitamin C.

In the present study, we adjusted the $12 \mathrm{~d}$ DR data into de-attenuated data to correct for day-to-day variations and energy-adjusted data to remove partial error related to energy intake. We found that the correlation coefficients between the de-attenuated and energy-adjusted FFQ data and the average of the $12 \mathrm{~d}$ DR showed an improvement for eight nutrients compared with the results obtained with the crude data. Similar improvements were previously reported after adjusting for total energy intake ${ }^{(44)}$, but contrasting results have also been reported ${ }^{(45,46)}$. The results may suggest that the variability of nutrient intake in the present study population was related to energy intake 
and therefore the adjustment for total energy intake using the residual method improved the correlation coefficients for some nutrients. Previous studies reported that energy adjustment decreased correlation coefficients when the variability depended on systematic errors of over/ underestimation $^{(47)}$.

We estimated calibration parameters by linear regression using $12 \mathrm{~d}$ DR and the FFQ to correct for quantitative differences in nutrient intake levels ${ }^{(48)}$. The calibration of the FFQ resulted in similar average intake estimations compared with the $12 \mathrm{~d}$ DR at the group level. Although calibration was needed to correct the mean nutrient intakes of the groups that were similar to those estimated by the $12 \mathrm{~d}$ DR, decreased standard deviations in the intake estimates from FFQ appeared for energy intake and intakes of most nutrients. Despite this shrinking tendency of standard deviations, which was also reported in an FFQ calibration study in Brazil ${ }^{(45)}$, a calibration factor is useful in correcting biases, particularly when dietary intake is the instrumental variable in a study of the association between diet and disease.

Several limitations need to be considered in the interpretation of our findings. First, both the DR and FFQ methods may have been biased with measurement errors, including erroneous recalls or changing diet, compared with other methods that use biomarkers such as doubly labelled water, urinary nitrogen and nutrient plasma concentration, which can be used in validation studies to improve the accuracy and objectivity of the results. Second, some relevant information including BMI, education levels, income and smoking status were not collected in the study; thus, we did not analyse the influence of these factors on the reproducibility and validity of the FFQ. Third, because participants were recruited in the Seoul metropolitan area only, selection bias may be present.

\section{Conclusion}

In conclusion, the 109-item interviewer-administered FFQ developed for assessing nutrient intake in the KNHANES has acceptable reproducibility and modest validity. The results indicate that the newly developed FFQ can be a useful dietary instrument to rank energy and nutrient intakes of Korean adults living in a metropolitan area. Further study is necessary to assess whether this FFQ produces similar results in other areas of Korea.

\section{Acknowledgements}

Financial support: The present study was supported by the Korea Centers for Disease Control and Prevention (grant number 2009E3301600). The Korea Centers for Disease Control and Prevention had no role in the design, analysis or writing of this article. Conflict of interest: None.
Authorship: The author's contributions are as follows. D.W.K. contributed to the data collection and data analysis and the interpretation of the study, and wrote the manuscript draft; S.S., J.S. and S.K. contributed to the data collection and data analysis; J.E.L., K.O. and H.Y.P. contributed to the conception and design of the study and the review of the manuscript; H.Y.P. contributed to the data interpretation and the review of the manuscript; H.J. contributed to the draft outline, data interpretation and manuscript review. All the authors read and approved the final manuscript. Ethics of buman subject participation: The study was conducted according to the guidelines in the Declaration of Helsinki and all procedures involving human subjects were approved by the Institutional Review Board of Seoul National University on 20 July 2009. Written informed consent was obtained from all participants.

\section{Supplementary material}

To view supplementary material for this article, please visit http://dx.doi.org/10.1017/S1368980014001712

\section{References}

1. Nishida C, Uauy R, Kumanyika S et al. (2004) The Joint WHO/FAO Expert Consultation on diet, nutrition and the prevention of chronic diseases: process, product and policy implications. Public Health Nutr 7, 245-250.

2. Kim S, Moon S \& Popkin BM (2000) The nutrition transition in South Korea. Am J Clin Nutr 71, 44-53.

3. Lee MJ, Popkin BM \& Kim S (2002) The unique aspects of the nutrition transition in South Korea: the retention of healthful elements in their traditional diet. Public Health Nutr 5, 197-203.

4. Kim YO, Kim MK, Lee SA et al. (2009) A study testing the usefulness of a dish-based food-frequency questionnaire developed for epidemiological studies in Korea. Br J Nutr 101, 1218-1227.

5. Resnicow K, Odom E, Wang T et al. (2000) Validation of three food frequency questionnaires and 24-hour recalls with serum carotenoid levels in a sample of AfricanAmerican adults. Am J Epidemiol 152, 1072-1080.

6. Kipnis V, Subar AF, Midthune D et al. (2003) Structure of dietary measurement error: results of the OPEN biomarker study. Am J Epidemiol 158, 14-21.

7. Subar AF, Kipnis V, Troiano RP et al. (2003) Using intake biomarkers to evaluate the extent of dietary misreporting in a large sample of adults: the OPEN study. Am J Epidemiol 158, $1-13$.

8. Basiotis PP, Welsh SO, Cronin FJ et al. (1987) Number of days of food intake records required to estimate individual and group nutrient intakes with defined confidence. $J$ Nutr 117, 1638-1641.

9. Ortiz-Andrellucchi A, Sánchez-Villegas A, Doreste-Alonso J et al. (2009) Dietary assessment methods for micronutrient intake in elderly people: a systematic review. Br J Nutr 102, Suppl. 1, S118-S149.

10. Ortiz-Andrellucchi A, Henríquez-Sánchez P, Sánchez-Villegas A et al. (2009) Dietary assessment methods for micronutrient intake in infants, children and adolescents: a systematic review. Br J Nutr 102, Suppl. 1, S87-S117.

11. Ortiz-Andrellucchi A, Doreste-Alonso J, Henríquez-Sánchez P et al. (2009) Dietary assessment methods for micronutrient 
intake in pregnant women: a systematic review. Br J Nutr 102, Suppl. 1, S64-S86.

12. Huybrechts I, De Bacquer D, Cox B et al. (2008) Variation in energy and nutrient intakes among pre-school children: implications for study design. Eur J Public Health $\mathbf{1 8}$ 509-516.

13. Oh SY \& Hong MH (1999) Within- and between-person variation of nutrient intakes of older people in Korea. Eur J Clin Nutr 53, 625-629.

14. Brinkman MT, Kellen E, Zeegers MP et al. (2011) Validation of the IMMIDIET food frequency questionnaire in an adult Belgian population: a report from the Belgian case-control study on bladder cancer risk. Acta Clin Belg 66, 18-25.

15. Dehghan M, Lopez Jaramillo P, Duenas R et al. (2011) Development and validation of a quantitative food frequency questionnaire among rural- and urban-dwelling adults in Colombia. J Nutr Educ Behav 44, 609-613.

16. Eng JY \& Moy FM (2011) Validation of a food frequency questionnaire to assess dietary cholesterol, total fat and different types of fat intakes among Malay adults. Asia Pac J Clin Nutr 20, 639-645.

17. Huang YC, Lee MS, Pan WH et al. (2011) Validation of a simplified food frequency questionnaire as used in the Nutrition and Health Survey in Taiwan (NAHSIT) for the elderly. Asia Pac J Clin Nutr 20, 134-140.

18. Mejia-Rodriguez F, Orjuela MA, Garcia-Guerra A et al. (2011) Validation of a novel method for retrospectively estimating nutrient intake during pregnancy using a semiquantitative food frequency questionnaire. Matern Child Health J 16, 1468-1483.

19. van Dongen MC, Lentjes MA, Wijckmans NE et al. (2011) Validation of a food-frequency questionnaire for Flemish and Italian-native subjects in Belgium: the IMMIDIET study. Nutrition 27, 302-309.

20. Molag ML, de Vries JH, Ocke MC et al. (2007) Design characteristics of food frequency questionnaires in relation to their validity. Am J Epidemiol 166, 1468-1478.

21. Korean Ministry of Health and Welfare (2006) National Health and Nutrition Examination Survey Report 2005. Seoul: Ministry of Health and Welfare (in Korean).

22. Korean Ministry of Health and Welfare (2010) Korea Health Statistics 2009: Korea National Health and Nutrition Examination Survey (KNHANES IV-3). Seoul: Korean Ministry of Health and Welfare (in Korean).

23. Yun SH, Shim JS, Kweon SH et al. (2013) Development of a food frequency questionnaire for the Korea National Health and Nutrition Examination Survey: data from the Fourth Korea National Health and Nutrition Examination Survey (KNHANES IV). Korean J Nutr 46, 186-196 (in Korean).

24. Korean National Academy of Agricultural Science (2007) Food Composition Table, 7th ed. Suwon: Rural Development Administration (in Korean).

25. Willett WC (1998) Nutritional Epidemiology. Oxford: Oxford University Press.

26. Willett WC, Sampson L, Stampfer MJ et al. (1985) Reproducibility and validity of a semiquantitative food frequency questionnaire. Am J Epidemiol 122, 51-65.

27. Johansson I, Hallmans G, Wikman A et al. (2002) Validation and calibration of food-frequency questionnaire measurements in the Northern Sweden Health and Disease cohort. Public Health Nutr 5, 487-496.

28. Villegas R, Yang G, Liu D et al. (2007) Validity and reproducibility of the food-frequency questionnaire used in the Shanghai men's health study. Br J Nutr 97, 993-1000.
29. Bland JM \& Altman DG (1986) Statistical methods for assessing agreement between two methods of clinical measurement. Lancet 1, 307-310.

30. Ambrosini GL, van Roosbroeck SAH, Mackerras D et al. (2003) The reliability of ten-year dietary recall: implications for cancer research. J Nutr 133, 2663-2668.

31. Marques-Vidal P, Ross A, Wynn E et al. (2011) Reproducibility and relative validity of a food-frequency questionnaire for French-speaking Swiss adults. Food Nutr Res 2011, 55.

32. Fernandez-Ballart JD, Pinol JL, Zazpe I et al. (2010) Relative validity of a semi-quantitative food-frequency questionnaire in an elderly Mediterranean population of Spain. Br J Nutr 103, 1808-1816.

33. Tsubono Y, Nishino Y, Fukao A et al. (1995) Temporal change in the reproducibility of a self-administered food frequency questionnaire. Am J Epidemiol 142, 1231-1235.

34. Cade J, Thompson R, Burley V et al. (2002) Development, validation and utilisation of food-frequency questionnaires a review. Public Health Nutr 5, 567-587.

35. Shahar DR, Yerushalmi N, Lubin F et al. (2001) Seasonal variations in dietary intake affect the consistency of dietary assessment. Eur J Epidemiol 17, 129-133.

36. Park MK, Noh HY, Song NY et al. (2012) Validity and reliability of a dish-based, semi-quantitative food frequency questionnaire for Korean diet and cancer research. Asia Pac J Cancer Prev 13, 545-552.

37. Hong S, Choi Y, Lee HJ et al. (2010) Development and validation of a semi-quantitative food frequency questionnaire to assess diets of Korean type 2 diabetic patients. Korean Diabetes J 34, 32-39.

38. Shim JS, Oh KW, Suh I et al. (2002) A study on validity of a semi-quantitative food frequency questionnaire for Korean adults. Korean J Community Nutr 7, 484-494.

39. Yang YJ, Kim MK, Hwang SH et al. (2010) Relative validities of 3-day food records and the food frequency questionnaire. Nutr Res Pract 4, 142-148.

40. Na YJ \& Lee SH (2012) Development and validation of a quantitative food frequency questionnaire to assess nutritional status in Korean adults. Nutr Res Pract 6, 444-450.

41. Ogawa K, Tsubono Y, Nishino Y et al. (2003) Validation of a food-frequency questionnaire for cohort studies in rural Japan. Public Health Nutr 6, 147-157.

42. Pietinen P, Hartman AM, Haapa E et al. (1988) Reproducibility and validity of dietary assessment instruments. II. A qualitative food frequency questionnaire. Am J Epidemiol 128, 667-676.

43. Torheim LE, Barikmo I, Hatloy A et al. (2001) Validation of a quantitative food-frequency questionnaire for use in Western Mali. Public Health Nutr 4, 1267-1277.

44. Subar AF, Thompson FE, Kipnis V et al. (2001) Comparative validation of the Block, Willett, and National Cancer Institute food frequency questionnaires: the Eating at America's Table Study. Am J Epidemiol 154, 1089-1099.

45. Araujo MC, Yokoo EM \& Pereira RA (2010) Validation and calibration of a semiquantitative food frequency questionnaire designed for adolescents. J Am Diet Assoc 110, 1170-1177.

46. Slater B, Philippi ST, Fisberg RM et al.. (2003) Validation of a semi-quantitative adolescent food frequency questionnaire applied at a public school in Sao Paulo, Brazil. Eur J Clin Nutr 57, 629-635.

47. Willett W \& Stampfer MJ (1986) Total energy intake: implications for epidemiologic analyses. Am J Epidemiol 124, 17-27.

48. Kaaks R, Riboli E \& van Staveren W (1995) Calibration of dietary intake measurements in prospective cohort studies. Am J Epidemiol 142, 548-556. 